\title{
Optimal estimation of group transformations using entanglement
}

\author{
G. Chiribella. . G. M. D'Ariano. fl and M. F. Sacchif \\ QUIT Quantum Information Theory Group of the INFM, Unità di Pavia \\ Dipartimento di Fisica "A. Volta", via Bassi 6, I-27100 Pavia, Italy
}

(Dated: October 1, 2018)

\begin{abstract}
We derive the optimal input states and the optimal quantum measurements for estimating the unitary action of a given symmetry group, showing how the optimal performance is obtained with a suitable use of entanglement. Optimality is defined in a Bayesian sense, as minimization of the average value of a given cost function. We introduce a class of cost functions that generalizes the Holevo class for phase estimation, and show that for states of the optimal form all functions in such a class lead to the same optimal measurement. A first application of the main result is the complete proof of the optimal efficiency in the transmission of a Cartesian reference frame. As a second application, we derive the optimal estimation of a completely unknown two-qubit maximally entangled state, provided that $N$ copies of the state are available. In the limit of large $N$, the fidelity of the optimal estimation is shown to be $1-3 /(4 N)$.

PACS numbers: 03.65.Ta, 03.67.-a
\end{abstract}

\section{INTRODUCTION}

Many of the most surprising advantages offered by the new technology of Quantum Information [1] arise from the concept of quantum entanglement. Computational speed-up [2, 3], quantum teleportation [4] and dense coding [5], secure protocols in cryptography $[6]$, precision enhancement in quantum measurements [7, 8 are just a short summary of some of the main lines of research inspired by entanglement.

After a so promising list, it is natural to expect remarkable improvements coming from entanglement also in the context of Quantum Estimation Theory [9, 10, in particular in the typical problem of estimating an unknown physical transformation drawn from a given set. With a heuristic argument inspired by dense coding, we could expect that the accuracy in the discrimination of a set of quantum channels can be increased by letting them act locally on a fixed side of a maximally entangled state. Even more, one is tempted to guess that a maximally entangled state is the optimal input for the estimation of an unknown black box. Even though these are both reasonable conjectures, in general they turn out to be false: for example, a maximally entangled input state is always useless - and often suboptimal - for the discrimination of two unitary transformations [7, 11]. The question then arises: is it really possible to make some general statement about the role of entanglement in the optimal estimation of an unknown transformation?

In this paper we will answer this question in the $c o$ -

\footnotetext{
*Electronic address: chiribella@unipv.it

$\dagger$ Electronic address: dariano@unipv.it Also at Center for Photonic Communication and Computing, Department of Electrical and Computer Engineering, Northwestern University, Evanston, IL 60208

${ }^{\ddagger}$ Electronic address: msacchi@unipv.it

$\S$ URL: http://www.qubit.it
}

variant case, which corresponds to the estimation of unitary transformations randomly picked out from a given representation of some group. To face the problem, we will choose the Bayesian approach, assuming a uniform a priori distribution for the unknown group parameters, and defining optimality as the minimization of the average value of a given cost function. Within the Bayesian framework, some results about the optimality of maximally entangled states have been presented in [7, 12 . Other results in the same direction have been derived in [13, 14] within a different approach based on quantum Cramér-Rao bound. However, all the mentioned results are limited to particular cases, and their extension to arbitrary representations of arbitrary groups is not straightforward.

Another nontrivial question is: which kind of entanglement is really useful for the estimation of group transformations? In Ref. [12], it has been considered the estimation of unitary transformations $U_{g}$ in $\mathbb{S U}(d)$ in the form $U_{g}^{\otimes N}$, corresponding to $N$ copies of the same unknown black box. The result is that the optimal performance can be attained by entangling the $N d$-level systems that undergo the unknown transformation with another set of $N d$-level systems playing the role of a reference system. However, as pointed out in Ref. 15, the entanglement with an additional set of $N$ reference systems actually is not needed: what really matters is something more subtle, namely the entanglement between spaces where the action of the group is irreducible and spaces where the action of the group is trivial. In the language of group theory, what is needed is maximal entanglement between representation spaces and multiplicity spaces. This kind of entanglement can be obtained not only by adding an external reference system as in [12], but also via the use of the multiple equivalent representations that appear in the Clebsch-Gordan decomposition of the representation $\left\{U_{g}^{\otimes N}\right\}$.

The concept of entanglement between representation spaces and multiplicity spaces will be the protagonist 
of this paper. In the following, we derive the optimal scheme for estimating an unknown group transformation, showing how this kind of entanglement allows to achieve the ultimate precision limits allowed by Quantum Mechanics. To do this, we introduce a class of cost functions that generalize the well known Holevo class for phase estimation [10], and show that all functions in such a class lead to the same optimal measurement. We give also an explicit expression for the average cost so that the optimization of the estimation scheme is reduced to a simple eigenvalue problem.

In Sec. III, before starting the analysis about optimal estimation strategies, we introduce the notation (II A) and some group theoretical tools (II B) that will be exploited throughout the paper. In Sec. III, we present the problem of estimating an unknown group transformation (III A), introducing a generalization to arbitrary groups of the Holevo class of cost functions (III B). The optimal input states are then derived (III C), and the entanglement between representation and multiplicity spaces is recognized to be the basic resource for an optimal estimation strategy. In order to find the optimal measurement for the estimation of a group transformation, we show in Sec. IIID how the special form of the optimal input states reflects on the covariance properties of the optimal measurement. Exploiting this analysis, we will show in IIIE that, for input states of the optimal form, all cost functions in the generalized Holevo class lead to the same optimal measurement. Finally, Sec. IV is devoted to applications of the general results. A first application (IV A) is the optimality proof of the protocol [15] for the absolute alignment of two reference frames. As a second application, we derive (IVB) the optimal estimation of a completely unknown two-qubit maximally entangled state with $N$ identical copies of the state. Section V concludes the paper, while the most technical proofs are provided in the Appendix.

\section{THEORETICAL TOOLS}

\section{A. Notation for bipartite states}

A simple notation can be introduced to deal with bipartite states. Given two Hilbert spaces $\mathcal{H}_{A}$ and $\mathcal{H}_{B}$, and fixed two orthonormal bases $\mathcal{B}_{A}=\left\{\left|\phi_{n}\right\rangle \mid n=1, \ldots, d_{A}\right\}$ and $\mathcal{B}_{B}=\left\{\left|\psi_{n}\right\rangle \mid n=1, \ldots, d_{B}\right\}$ for $\mathcal{H}_{A}$ and $\mathcal{H}_{B}$ respectively, it is possible to associate in a one to one way any vector $|C\rangle\rangle \in \mathcal{H}_{A} \otimes \mathcal{H}_{B}$ with an operator $C \in \mathcal{B}\left(\mathcal{H}_{B}, \mathcal{H}_{A}\right)$ via the relation [16]

$$
|C\rangle\rangle=\sum_{m, n}\left\langle\phi_{m}|C| \psi_{n}\right\rangle\left|\phi_{m}\right\rangle\left|\psi_{n}\right\rangle .
$$

With this notation, one has the simple relations

$$
\langle\langle C \mid D\rangle\rangle=\operatorname{Tr}\left[C^{\dagger} D\right]
$$

and

$$
\left.A \otimes B|C\rangle\rangle=\left|A C B^{T}\right\rangle\right\rangle
$$

for any $A \in \mathcal{B}\left(\mathcal{H}_{A}\right)$ and $B \in \mathcal{B}\left(\mathcal{H}_{B}\right)$, where transposition $T$ is defined with respect to the fixed bases. Such relations allow to greatly simplify the calculation involving entangled states, and will be extensively used throughout the paper.

\section{B. Elements of group theory}

Here we recall some simple tools of group theory [17] that will be exploited throughout the paper.

Suppose we are given a Hilbert space $\mathcal{H}$ and a unitary representation $\mathrm{R}(\mathbf{G})=\left\{U_{g} \in \mathcal{B}(\mathcal{H}) \mid g \in \mathbf{G}\right\}$ of a compact Lie group $\mathbf{G}$. The Hilbert space can be decomposed into orthogonal subspaces in the following way

$$
\mathcal{H} \equiv \bigoplus_{\mu \in \mathrm{S}} \mathcal{H}_{\mu} \otimes \mathbb{C}^{m_{\mu}}
$$

where the sum runs over the set of irreducible representations of $\mathbf{G}$ that appear in the Clebsch-Gordan decomposition of $\mathrm{R}(\mathbf{G})$. The action of the group is irreducible in each representation space $\mathcal{H}_{\mu}$, while it is trivial in the multiplicity space $\mathbb{C}^{m_{\mu}}$, namely

$$
U_{g} \equiv \bigoplus_{\mu \in \mathrm{S}} U_{g}^{\mu} \otimes \mathbb{1}_{m_{\mu}}
$$

$\mathbb{1}_{d}$ denoting the identity in a $d$-dimensional Hilbert space. The projection $\Pi_{\mu}$ onto the subspace $\mathcal{H}_{\mu} \otimes \mathbb{C}^{m_{\mu}}$ is given by the integral formula

$$
\Pi_{\mu}=d_{\mu} \int \mathrm{d} g \chi^{\mu *}(g) U_{g},
$$

where $\mathrm{d} g$ denotes the normalized invariant Haar measure $(\mathrm{d} g=\mathrm{d}(k g)=\mathrm{d}(g k)$ for any $k, g \in \mathbf{G}), d_{\mu} \equiv$ $\operatorname{dim}\left(\mathcal{H}_{\mu}\right)$, and $\chi^{\mu}(g) \equiv \operatorname{Tr}\left[U_{g}^{\mu}\right]$ is the character of the irreducible representation $\mu$. Note that here we are considering $\mathbf{G}$ as a continuous group only for fixing notation, nevertheless - here and all throughout the paper - $\mathbf{G}$ can have a finite number of elements, say $|\mathbf{G}|$, and in this case we have simply to replace integrals with sums and d $g$ with $1 /|\mathbf{G}|$.

Moreover, any operator $O \in \mathcal{B}(\mathcal{H})$ in the commutant of $\mathrm{R}(\mathbf{G})$-i.e. such that $\left[O, U_{g}\right]=0 \quad \forall g \in \mathbf{G}$ - has the form

$$
O=\bigoplus_{\mu \in \mathrm{S}} \mathbb{1}_{d_{\mu}} \otimes O_{\mu}
$$

where $O_{\mu}$ is a $m_{\mu} \times m_{\mu}$ complex matrix. In particular, the group average $\langle A\rangle_{\mathbf{G}} \equiv \int \mathrm{d} g U_{g} A U_{g}^{\dagger}$ of a given operator $A$ with respect to the invariant Haar measure is in the commutant of $\mathrm{R}(\mathbf{G})$, and has the form:

$$
\langle A\rangle_{\mathbf{G}}=\bigoplus_{\mu \in \mathbf{S}} \mathbb{1}_{d_{\mu}} \otimes \frac{1}{d_{\mu}} \operatorname{Tr}_{\mathcal{H}_{\mu}}[A],
$$


where $\operatorname{Tr}_{\mathcal{H}_{\mu}}[A]$ is a short notation for $\operatorname{Tr}_{\mathcal{H}_{\mu}}\left[\Pi_{\mu} A \Pi_{\mu}\right], \Pi_{\mu}$ being the projection onto $\mathcal{H}_{\mu} \otimes \mathbb{C}^{m_{\mu}}$. Here and throughout the paper we assume the normalization of the Haar measure: $\int_{\mathbf{G}} \mathrm{d} g=1$.

Remark I: entanglement between representation spaces and multiplicity spaces.

The choice of an orthonormal basis $\mathrm{B}^{\mu}=\left\{\left|\phi_{n}^{\mu}\right\rangle \in \mathbb{C}^{m_{\mu}} \mid n=1, \ldots, m_{\mu}\right\}$ for a multiplicity space fixes a particular decomposition of the Hilbert space as a direct sum of irreducible subspaces:

$$
\mathcal{H}_{\mu} \otimes \mathbb{C}^{m_{\mu}}=\oplus_{n=1}^{m_{\mu}} \mathcal{H}_{n}^{\mu},
$$

where $\mathcal{H}_{n}^{\mu} \equiv \mathcal{H}_{\mu} \otimes\left|\phi_{n}^{\mu}\right\rangle$. In this picture, it is clear that $m_{\mu}$ is the number of different irreducible subspaces carrying the same representation $\mu$, each of them having dimension $d_{\mu}$. Moreover, with respect to the decomposition (㺼), any pure state $|\Psi\rangle \in \mathcal{H}$ can be written as

$$
\left.|\Psi\rangle=\bigoplus_{\mu \in \mathrm{S}} c_{\mu}\left|\Psi_{\mu}\right\rangle\right\rangle
$$

where $\left.\left|\Psi_{\mu}\right\rangle\right\rangle$ is a bipartite state in $\mathcal{H}_{\mu} \otimes \mathbb{C}^{m_{\mu}}$ and $\sum_{\mu \in \mathrm{S}}\left|c_{\mu}\right|^{2}=1$. With respect to the direct sum decomposition (9), the Schmidt number of such a state is the minimum number of subspaces carrying the same representation $\mu \in \mathrm{S}$ that are needed to decompose $|\Psi\rangle$.

Remark II: maximum number of equivalent representations in the decomposition of a pure state.

The Schmidt number of any bipartite state $\left.\left|\Psi_{\mu}\right\rangle\right\rangle \in \mathcal{H}_{\mu} \otimes \mathbb{C}^{m_{\mu}}$ is always less then or equal to $k_{\mu}=\min \left\{d_{\mu}, m_{\mu}\right\}$. This means that any pure state can be decomposed using no more than $k_{\mu}$ irreducible subspaces carrying the same representation $\mu \in \mathrm{S}$.

\section{OPTIMAL ESTIMATION OF GROUP TRANSFORMATIONS}

\section{A. Background problem}

Suppose we are given a black box that performs on a system $\mathcal{S}$ an unknown unitary transformation $U_{g}$ randomly drawn from a group representation $\mathrm{R}(\mathbf{G})$. In order to estimate the transformation $U_{g}$, we can prepare the system in an input state $\rho^{\mathcal{S}}$, send it through the black box, and try to estimate the parameter $g$ from the output state

$$
\rho_{g}^{\mathcal{S}} \equiv U_{g} \rho^{\mathcal{S}} U_{g}^{\dagger} .
$$

More generally, we can also exploit an additional reference system $\mathcal{R}$ and prepare an entangled state $\rho^{\mathcal{S R}}$, so that the output state becomes

$$
\rho_{g}^{\mathcal{S R}} \equiv\left(U_{g} \otimes \mathbb{1}_{\mathcal{R}}\right) \rho^{\mathcal{S R}}\left(U_{g}^{\dagger} \otimes \mathbb{1}_{\mathcal{R}}\right) .
$$

Our task is to find the best input states and the best estimation strategies allowed by Quantum Mechanics to determine the parameter $g$. Since we are interested in ultimate in-principle limits, we assume complete freedom in preparing any physical state and in realizing any quantum measurement. This means that we are allowed to choose the state $\rho^{\mathcal{S} \mathcal{R}}$ with minimal stability group, reducing the set of unitaries that are not discriminable to those that differ just by a phase factor. Therefore, the stability group can be only a (nontrivial) center for $\mathbf{G}$, made of multiples of the identity, corresponding to (a subgroup of) $U(1)$. The quotient group is then a group itself, and in the following we will use the same symbol $\mathbf{G}$ for such a quotient group. Notice that the requirement of central stability group $U(1)$ is satisfied by choosing the state $\rho^{\mathcal{S R}}$ as pure, and with maximal Schmidt number.

The most general estimating strategy allowed by quantum mechanics, including both quantum measurements and classical data processing, can be described by a Positive Operator Valued Measure (POVM) $M$ that associates to any estimate $\hat{g} \in \mathbf{G}$ a positive semidefinite operator $M(\hat{g})$, satisfying the normalization condition

$$
\int_{\mathbf{G}} \mathrm{d} g M(g)=\mathbb{1} .
$$

The probability density of the estimate $\hat{g}$ in the state $\rho_{g}$ is given by the usual Born rule:

$$
p(\hat{g} \mid g)=\operatorname{Tr}\left[\rho_{g} M(\hat{g})\right] .
$$

In this paper, the estimation problem will be faced in the Bayesian setting with prior uniform probability density $\mathrm{d} g$, and the optimal estimation will be defined as the one that minimizes the average value of a given cost function $c(\hat{g}, g)$ that associates to any estimate $\hat{g}$ a cost which increases versus the "distance" of $\hat{g}$ from the true value $g$. The average of the cost function over the prior and the conditional probability distributions will be given by

$$
\langle c\rangle=\int \mathrm{d} g \int \mathrm{d} \hat{g} c(\hat{g}, g) p(\hat{g} \mid g) .
$$

\section{B. A generalized Holevo class of cost functions}

We will make two assumptions on the form of the cost function $c(\hat{g}, g)$.

First assumption. We require $c$ to be group invariant, namely

$$
c(\hat{g}, g)=c(k \hat{g}, k g) \quad \forall \hat{g}, g, k \in \mathbf{G}
$$

(left-invariance), and

$$
c(\hat{g}, g)=c(g k, \hat{g} k) \quad \forall \hat{g}, g, k \in \mathbf{G}
$$

(right-invariance). By using Fourier analysis, one can prove (see Appendix) that this assumption is equivalent 
to the expansion

$$
c(\hat{g}, g)=\sum_{\sigma} a_{\sigma} \chi^{\sigma *}\left(\hat{g} g^{-1}\right),
$$

where $\chi^{\sigma}(g) \equiv \operatorname{Tr}\left[U^{\sigma}(g)\right]$ is the character of the irreducible representation $\sigma$, and the coefficients $a_{\sigma}$ satisfy the identity $a_{\sigma}^{*}=a_{\sigma *} \forall \sigma$, in order to have a real cost function.

Second assumption. We require all nonzero coefficients $a_{\sigma}$ in the expression (18) to be negative, with the only exception of the coefficient $a_{\sigma_{0}}$ corresponding to the trivial representation $U^{\sigma_{0}}(g)=1 \quad \forall g$, which is allowed to be positive (the $\sigma_{0}$ term just adds a trivial constant to the cost function, since $\left.\chi^{\sigma_{0}}(g)=1 \quad \forall g\right)$.

The class of functions that satisfy our two assumptions is a direct generalization of the class of cost functions introduced by Holevo for the estimation of phase shifts [10]. In fact, such functions have the form

$$
c(\hat{\phi}-\phi)=\sum_{k \in \mathbb{Z}} a_{k} e^{-i k(\hat{\phi}-\phi)},
$$

where $a_{k} \leq 0$ for any $k \neq 0$, and $e^{i k \phi}$ is the character of the unidimensional representation labeled by $k$.

\section{Optimal choice of the input state}

Since the average cost (15) is a linear functional of the input state $\rho$, in the optimization problem we can restrict attention to pure input states $\rho=|\Psi\rangle\langle\Psi|$. Then the problem becomes equivalent to the optimal discrimination problem of states in the orbit

$$
\mathcal{O}=\left\{\left|\Psi_{g}\right\rangle \equiv U_{g}|\Psi\rangle \mid g \in \mathbf{G}\right\}
$$

generated from $|\Psi\rangle$ by the action of the representation $\mathrm{R}(\mathbf{G})$.

Let's consider the Clebsch-Gordan decomposition (5) of the unitaries $U_{g}$. From now on we will assume the algebraic condition

$$
m_{\mu}=d_{\mu} \quad \forall \mu \in \mathrm{S} .
$$

Lemma 1 The assumption (21) can be done without any loss of generality.

Proof. Suppose $d_{\mu}>m_{\mu}$ for some representation $\mu$. In this case, we can introduce a reference system $\mathcal{R}$ whose dimension is

$$
d_{\mathcal{R}} \geq \max _{\mu \in \mathrm{S}}\left\{\frac{d_{\mu}}{m_{\mu}}\right\},
$$

and replace $U_{g}$ with its extension $U_{g}^{\prime}=U_{g} \otimes \mathbb{1}_{\mathcal{R}}$, acting in the tensor product Hilbert space $\mathcal{H} \otimes \mathcal{H}_{\mathcal{R}}$. In this way, $U_{g}^{\prime}$ will satisfy the condition $m_{\mu}^{\prime} \equiv m_{\mu} \times d_{\mathcal{R}} \geq d_{\mu} \quad \forall \mu$. On the other hand, as already mentioned at the end of Sec. IIB, any pure state $|\Psi\rangle$ can be decomposed in the form (10) with no more than $k_{\mu}=\min \left\{d_{\mu}, m_{\mu}\right\}$ irreducible subspaces for any $\mu$. Therefore, we can switch our attention from the whole Hilbert space $\mathcal{H} \otimes \mathcal{H}_{\mathcal{R}}=\bigoplus_{\mu} \mathcal{H}_{\mu} \otimes$ $\mathbb{C}^{m_{\mu}^{\prime}}$ to the invariant subspace $\mathcal{H}^{\prime} \equiv \bigoplus_{\mu} \mathcal{H}_{\mu} \otimes \mathbb{C}^{d_{\mu}}$, which contains the input state $|\Psi\rangle$ along with its orbit (20). In other words, without loss of generality we can always consider an input state in the Hilbert space

$$
\mathcal{H}^{\prime}=\bigoplus_{\mu} \mathcal{H}_{\mu} \otimes \mathbb{C}^{d_{\mu}}
$$

which can be thought as embedded in a larger Hilbert space $\mathcal{H} \otimes \mathcal{H}_{\mathcal{R}}$.

Remark. The need of adding an external reference system $\mathcal{R}$ arises only in the case when $d_{\mu}>m_{\mu}$ for some irreducible representation $\mu$. In fact, the role of the reference system is simply to increase the number of equivalent representations until the extended Hilbert space $\mathcal{H} \otimes \mathcal{H}_{\mathcal{R}}$ reaches the threshold $m_{\mu} \geq d_{\mu} \quad \forall \mu$. This observation allows to greatly reduce the dimension of the reference system with respect to the customary estimation schemes inspired by dense coding, with a reference system $\mathcal{H}_{\mathcal{R}}$ having the same dimension of $\mathcal{H}$.

Now we show that the best input state $|\Psi\rangle$ for estimating the group transformation of an unknown black box is a state of the form (10), with each $\left.\left|\Psi_{\mu}\right\rangle\right\rangle$ maximally entangled, namely

$$
\left.\left|\Psi_{\mu}\right\rangle\right\rangle=\frac{1}{\sqrt{d_{\mu}}} \sum_{n=1}^{d_{\mu}}\left|\psi_{n}^{\mu}\right\rangle\left|\phi_{n}^{\mu}\right\rangle,
$$

$\mathrm{B}_{A}^{\mu}=\left\{\left|\psi_{n}^{\mu}\right\rangle \mid n=1, \ldots, d_{\mu}\right\}$ and $\mathrm{B}_{B}^{\mu}=\left\{\left|\phi_{n}^{\mu}\right\rangle \mid n=\right.$ $\left.1, \ldots, d_{\mu}\right\}$ being Schmidt bases for $\mathcal{H}_{\mu}$ and $\mathbb{C}^{d_{\mu}}$ respectively. Exploiting the notation (1) - with fixed bases $\mathrm{B}_{A}^{\mu}$ and $\mathrm{B}_{B}^{\mu}$ - the optimal input state $|\Psi\rangle$ must have the form

$$
\left.|\Psi\rangle=\bigoplus_{\mu \in \mathrm{S}} \frac{c_{\mu}}{\sqrt{d_{\mu}}}\left|W_{\mu}\right\rangle\right\rangle,
$$

with $W_{\mu} \equiv \sum_{n}\left|\psi_{n}^{\mu}\right\rangle\left\langle\phi_{n}^{\mu}\right|$ unitary operators.

Theorem 1 (optimal input states) With a suitable choice of the coefficients $\left\{c_{\mu}\right\}$, any input state of the form (25) achieves the minimum average cost.

Suppose that the minimum cost $\langle c\rangle^{O p t}$ is achieved by the input state $\left.|\Phi\rangle=\bigoplus_{\mu} c_{\mu}\left|\Phi_{\mu}\right\rangle\right\rangle$ along with the estimation strategy described by the POVM $M(g)$. The operator $K_{h} \equiv \bigoplus_{\mu} \mathbb{1}_{\mu} \otimes \sqrt{d_{\mu}}\left(W_{\mu}^{\dagger} U_{h}^{\mu} \Phi_{\mu}\right)^{T}$ converts the orbit of an input state 25) into the orbit of the optimal input state $|\Phi\rangle$, since using identity $(\beta)$, we have

$$
K_{h}\left|\Psi_{g}\right\rangle=\left|\Phi_{g h}\right\rangle
$$

where $\left|\Psi_{g}\right\rangle=U_{g}|\Psi\rangle$ and $\left|\Phi_{g}\right\rangle=U_{g}|\Phi\rangle$. Consider now the POVM $M^{\prime}(g) \equiv \int \mathrm{d} h K_{h}^{\dagger} M(g h) K_{h}$. The POVM 
$M^{\prime}(g)$ is normalized, since

$$
\begin{aligned}
\int \mathrm{d} g M^{\prime}(g) & =\int \mathrm{d} g \int \mathrm{d} h K_{h}^{\dagger} M(g h) K_{h} \\
& =\int \mathrm{d} h K_{h}^{\dagger} K_{h} \\
& =\mathbb{1},
\end{aligned}
$$

where we exchanged integrals over $g$ and $h$, used invariance of the Haar measure $\mathrm{d} g$, and finally used Eq. (8) and the normalization of bipartite states $\left.\left|\Phi_{\mu}\right\rangle\right\rangle$ in the form $\operatorname{Tr}\left[\Phi_{\mu}^{\dagger} \Phi_{\mu}\right]=1$. A state $|\Psi\rangle$ of the form (25) along with the POVM $M^{\prime}(g)$ achieves the minimum cost. In fact,

$$
\begin{aligned}
\langle c\rangle & =\int \mathrm{d} g \int \mathrm{d} \hat{g} c(\hat{g}, g)\left\langle\Psi_{g}\left|M^{\prime}(\hat{g})\right| \Psi_{g}\right\rangle \\
& =\int \mathrm{d} g \int \mathrm{d} \hat{g} \int \mathrm{d} h c(\hat{g}, g)\left\langle\Phi_{g h}|M(\hat{g} h)| \Phi_{g h}\right\rangle \\
& =\int \mathrm{d} g \int \mathrm{d} \hat{g} \int \mathrm{d} h c(\hat{g} h, g h)\left\langle\Phi_{g h}|M(\hat{g} h)| \Phi_{g h}\right\rangle \\
& =\int \mathrm{d} k \int \mathrm{d} \hat{k} c(\hat{k}, k)\left\langle\Phi_{k}|M(\hat{k})| \Phi_{k}\right\rangle \\
& =\langle c\rangle^{O p t},
\end{aligned}
$$

where we used right-invariance of both cost function and Haar measure.

\section{Covariance properties of the estimating POVM}

Since the whole orbit $(20)$ is generated from the input state $|\Psi\rangle$ by the action $\mathrm{R}(\mathbf{G})$ of the group, there is no loss of generality in restricting attention to estimating POVM of the covariant form [10

$$
M(g)=U_{g} \Xi U_{g}^{\dagger}
$$

with $\Xi$ a suitable positive operator satisfying the normalization condition (13). A covariant POVM yields a left-invariant probability distribution, namely $p(k \hat{g} \mid k g)=$ $p(\hat{g} \mid g) \quad \forall k, \hat{g}, g \in \mathbf{G}$. Using both the left-invariance of the probability distribution and of the cost function, the average cost (15) can be written as

$$
\langle c\rangle=\int \mathrm{d} g c(g, e) p(g \mid e)
$$

where $e$ is the identity element of the group $\mathbf{G}$.

For superpositions of maximally entangled states as in Eq. (25), the orbit $\mathcal{O}$ enjoys an additional symmetry that reflects on an additional covariance property of the POVM. In fact, using the decomposition (5) and the identity (3), we can note that

$$
\begin{aligned}
\left|\Psi_{g}\right\rangle & =U_{g}|\Psi\rangle \\
& \left.=\bigoplus_{\mu \in \mathcal{S}} \frac{c_{\mu}}{\sqrt{d_{\mu}}}\left(U_{g}^{\mu} \otimes \mathbb{1}_{\mu}\right)\left|W_{\mu}\right\rangle\right\rangle \\
& \left.=\bigoplus_{\mu \in \mathcal{S}} \frac{c_{\mu}}{\sqrt{d_{\mu}}}\left[\mathbb{1}_{\mu} \otimes\left(W_{\mu}^{\dagger} U_{g}^{\mu} W_{\mu}\right)^{T}\right]\left|W_{\mu}\right\rangle\right\rangle \\
& =V_{g}^{\dagger}|\Psi\rangle \quad \forall g \in \mathbf{G},
\end{aligned}
$$

where

$$
V_{g} \equiv \oplus_{\mu \in \mathrm{S}}\left(\mathbb{1}_{\mu} \otimes\left(W_{\mu}^{\dagger} U_{g}^{\mu} W_{\mu}\right)^{*}\right),
$$

is an element of a new unitary representation $R^{\prime}(\mathbf{G})$ of the group $\mathbf{G}$. Notice that the two representations $R(\mathbf{G})$ and $\mathrm{R}^{\prime}(\mathbf{G})$ commute among themselves. Then, the following Lemma holds:

Lemma 2 There is no loss of generality in assuming a covariant POVM $M(g)=U_{g} \Xi U_{g}^{\dagger}$ with

$$
\left[\Xi, U_{g} V_{g}\right]=0 \quad \forall g \in \mathbf{G},
$$

where $U_{g}$ and $V_{g}$ are given in Eqs. (5) and (29), respectively.

Proof. For any possible POVM $N(g)$ there is a covariant POVM with the above property and with the same average cost. In fact, the group average

$$
M(g)=\int \mathrm{d} k \int \mathrm{d} h U_{k}^{\dagger} V_{h}^{\dagger} N\left(k g h^{-1}\right) V_{h} U_{k}
$$

is covariant-namely $M(g)=U_{g} \Xi U_{g}^{\dagger}$ with $\Xi=M(e)$ and satisfies the required commutation relation (30). Both properties follow simply from the invariance of the Haar measure. To prove that the cost of the covariant POVM $M(g)$ is the same as the cost of $N(g)$ we use the property

$$
U_{k} V_{h}\left|\Psi_{g}\right\rangle=\left|\Psi_{k g h^{-1}}\right\rangle \quad \forall k, h, g \in \mathbf{G}
$$

of the states generated from the input (25). In this way,

$$
\begin{aligned}
\langle c\rangle_{M} & \equiv \int \mathrm{d} g \int \mathrm{d} \hat{g} c(\hat{g}, g)\left\langle\Psi_{g}|M(\hat{g})| \Psi_{g}\right\rangle \\
& =\int \mathrm{d} g \int \mathrm{d} \hat{g} \int \mathrm{d} k \int \mathrm{d} h c(\hat{g}, g) \times \\
\times\left\langle\Psi_{k g h^{-1}}\left|N\left(k \hat{g} h^{-1}\right)\right| \Psi_{k g h^{-1}}\right\rangle & \\
& =\int \mathrm{d} g \int \mathrm{d} \hat{g} \int \mathrm{d} k \int \mathrm{d} h c\left(k \hat{g} h^{-1}, k g h^{-1}\right) \times \\
& \times\left\langle\Psi_{k g h^{-1}}\left|N\left(k \hat{g} h^{-1}\right)\right| \Psi_{k g h^{-1}}\right\rangle \\
& \equiv\langle c\rangle_{N},
\end{aligned}
$$

where we used the left- and right-invariance of the cost function $c(\hat{g}, g)$.

Let's diagonalize the operator $\Xi$ and express its (non-

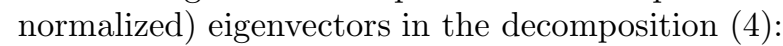

$$
\begin{aligned}
\Xi & =\sum_{i=1}^{r}\left|\eta^{i}\right\rangle\left\langle\eta^{i}\right| \\
& \left.=\sum_{i} \bigoplus_{\mu, \nu} \sqrt{d_{\mu} d_{\nu}}\left|\eta_{\mu}^{i}\right\rangle\right\rangle\left\langle\left\langle\eta_{\nu}^{i}\right|,\right.
\end{aligned}
$$

where the factor $\sqrt{d_{\mu}}$ has been inserted just for later convenience. 
Lemma 3 Any covariant POVM $M(g)=U_{g} \Xi U_{g}^{\dagger}$ with the commutation property (30) must satisfy the two relations:

$$
\sum_{i} \eta_{\mu}^{i \dagger} \eta_{\mu}^{i}=\mathbb{1}_{\mu} \quad \forall \mu \in \mathrm{S}
$$

and

$$
\sum_{i} \eta_{\mu}^{i} \eta_{\mu}^{i \dagger}=\mathbb{1}_{\mu} \quad \forall \mu \in \mathrm{S}
$$

Proof. The normalization (13) becomes

$$
\langle\Xi\rangle_{\mathbf{G}} \doteq \int \mathrm{d} g U_{g} \Xi U_{g}^{\dagger}=\mathbb{1} .
$$

The group average $\langle\Xi\rangle_{\mathbf{G}}$ can be expressed using Eq. (8). In this way, Eq. (36) becomes

$$
\frac{1}{d_{\mu}} \operatorname{Tr}_{\mathcal{H}_{\mu}}[\Xi]=\mathbb{1}_{\mu} \quad \forall \mu \in \mathrm{S} .
$$

By explicit computation,

$$
\begin{aligned}
\frac{1}{d_{\mu}} \operatorname{Tr}_{\mathcal{H}_{\mu}}[\Xi] & =\sum_{i} \operatorname{Tr}_{\mathcal{H}_{\mu}}\left[\left|\eta_{\mu}^{i}\right\rangle\right\rangle\left\langle\left\langle\eta_{\mu}^{i}\right|\right] \\
& =\sum_{i} \eta_{\mu}^{i T} \operatorname{Tr}_{\mathcal{H}_{\mu}}\left[\left|\mathbb{1}_{\mu}\right\rangle\right\rangle\left\langle\left\langle\mathbb{1}_{\mu}\right|\right] \eta_{\mu}^{i *} \\
& =\sum_{i} \eta_{\mu}^{i T} \eta_{\mu}^{i *} .
\end{aligned}
$$

Substituting this expression in (37) and taking the complex conjugate we get (34). Moreover, using the commutation relation (30), we can transform the group average with respect to $R(\mathbf{G})$ in a group average with respect to $\mathrm{R}^{\prime}(\mathbf{G})$, namely

$$
\begin{aligned}
\langle\Xi\rangle_{\mathbf{G}} & =\int \mathrm{d} g U_{g}\left(U_{g}^{\dagger} V_{g}^{\dagger} \Xi U_{g} V_{g}\right) U_{g}^{\dagger} \\
& =\int \mathrm{d} g V_{g}^{\dagger} \Xi V_{g} .
\end{aligned}
$$

In this way, using Eq. (29), Eq. (35) can be proved along the same lines used to prove Eq. (34).

\section{E. The optimal POVM}

We are now able to find the optimal covariant POVM for the estimation of group transformation with superpositions of maximally entangled states.

Theorem 2 (optimal POVM) In the estimation of the states in the orbit $\mathcal{O}$ generated from the input state

$$
\left.|\Psi\rangle=\bigoplus_{\mu \in \mathrm{S}} \frac{c_{\mu}}{\sqrt{d_{\mu}}}\left|W_{\mu}\right\rangle\right\rangle
$$

where $W_{\mu}$ are unitary operators, the covariant POVM given by $\Xi=|\eta\rangle\langle\eta|$ with

$$
\left.|\eta\rangle=\bigoplus_{\mu \in \mathrm{S}} \sqrt{d_{\mu}} e^{i \arg \left(c_{\mu}\right)}\left|W_{\mu}\right\rangle\right\rangle
$$

is optimal for any cost function $c(\hat{g}, g)$ of the form

$$
c(\hat{g}, g)=\sum_{\sigma} a_{\sigma} \chi^{\sigma *}\left(\hat{g} g^{-1}\right),
$$

with $a_{\sigma} \leq 0 \quad \forall \sigma \neq \sigma_{0}$.

The average cost corresponding to the optimal estimation strategy is

$$
\langle c\rangle^{O p t}=a_{\sigma_{0}}+\sum_{\mu, \nu}\left|c_{\mu}\right| C_{\mu \nu}\left|c_{\nu}\right|,
$$

where

$$
C_{\mu \nu} \equiv \sum_{\sigma \neq \sigma_{0}} a_{\sigma} m_{\sigma}^{(\mu \nu)}
$$

$m_{\sigma}^{(\mu \nu)}$ being the multiplicity of the irreducible representation $\sigma$ in the Clebsch-Gordan series of the tensor product $U_{g}^{\mu} \otimes U_{g}^{\nu *}$.

Proof. We will show that Eq. (41) gives a lower bound for the average cost, and that the POVM $\Xi=|\eta\rangle\langle\eta|$ with $|\eta\rangle$ given by Eq. (39) achieves this bound. By using identities (2) and (3), and the form (33) for the operator $\Xi$, Eq. (28) becomes

$$
\begin{aligned}
\langle c\rangle= & \int \mathrm{d} g c(g, e) \times \\
& \times \sum_{i} \sum_{\mu, \nu} c_{\mu}^{*} c_{\nu} \operatorname{Tr}\left[W_{\mu}^{\dagger} U_{g}^{\mu} \eta_{\mu}^{i} \otimes W_{\nu}^{T} U_{g}^{\nu *} \eta_{\nu}^{i *}\right] .
\end{aligned}
$$

Let's expand $c(g, e)$ as in (40). Subtracting from the average cost $\langle c\rangle$ the constant term $a_{\sigma_{0}}$, which is not relevant for the optimization, we get

$$
\begin{aligned}
\langle c\rangle-a_{\sigma_{0}}= & \sum_{i} \sum_{\mu, \nu} c_{\mu}^{*} c_{\nu} \times \\
& \times \sum_{\sigma \neq \sigma_{0}} \frac{a_{\sigma}}{d_{\sigma}} \operatorname{Tr}\left[\Pi_{\sigma}^{(\mu \nu)}\left(\eta_{\mu}^{i} W_{\mu}^{\dagger} \otimes \eta_{\nu}^{i *} W_{\nu}^{T}\right)\right],
\end{aligned}
$$

where we defined

$$
\Pi_{\sigma}^{(\mu \nu)} \equiv d_{\sigma} \int \mathrm{d} g \chi^{\sigma *}(g) U_{g}^{\mu} \otimes U_{g}^{\nu *} .
$$

According to $(\bar{\Phi}), \Pi_{\sigma}^{(\mu \nu)}$ is the projection onto the direct sum of all the subspaces of $\mathcal{H}_{\mu} \otimes \mathcal{H}_{\nu}$ that carry the irreducible representation $\sigma$ in the tensor product $U_{g}^{\mu} \otimes U_{g}^{\nu *}$. Clearly $\Pi_{\sigma}^{(\mu \nu)}$ is nonzero if and only if the Clebsch-Gordan series of $U_{g}^{\mu} \otimes U_{g}^{\nu^{*}}$ contains $\sigma$ with nonzero multiplicity $m_{\sigma}^{(\mu \nu)}$. Notice also that $\operatorname{Tr}\left[\Pi_{\sigma}^{(\mu \nu)}\right]=d_{\sigma} m_{\sigma}^{(\mu \nu)}$, by definition of $\Pi_{\sigma}^{(\mu \nu)}$. 
Denoting by $\sum_{\mu, \nu, \sigma}^{\prime}$ the sum over $\mu, \nu$ and all $\sigma$ except $\sigma_{0}$, the average cost can be bounded as follows

$$
\begin{aligned}
\langle c\rangle-a_{\sigma_{0}} & \geq \sum_{\mu, \nu, \sigma}^{\prime} \frac{a_{\sigma}}{d_{\sigma}} \mid c_{\mu} c_{\nu} \sum_{i} \operatorname{Tr}\left[\Pi_{\sigma}^{(\mu \nu)}\left(\eta_{\mu}^{i} W_{\mu}^{\dagger} \otimes \eta_{\nu}^{i *} W_{\nu}^{T}\right)\right] \\
& \geq \sum_{\mu, \nu, \sigma}^{\prime} \frac{a_{\sigma}}{d_{\sigma}}\left|c_{\mu} c_{\nu}\right| \sqrt{\left(\sum_{i} \operatorname{Tr}\left[\Pi_{\sigma}^{(\mu \nu)}\left(\eta_{\mu}^{i} \eta_{\mu}^{i \dagger} \otimes \mathbb{1}_{\nu}\right)\right]\right)} \\
& \times \sqrt{\left(\sum_{j} \operatorname{Tr}\left[\Pi_{\sigma}^{(\mu \nu)}\left(\mathbb{1}_{\mu} \otimes W_{\mu}^{*} \eta_{\nu}^{j T} \eta_{\nu}^{j *} W_{\mu}^{T}\right)\right]\right)}
\end{aligned}
$$

since all $a_{\sigma}$ are nonpositive. The second inequality follows from Cauchy-Schwartz inequality with respect to the scalar product $\langle\mathbf{A}, \mathbf{B}\rangle \equiv \sum_{i} \operatorname{Tr}\left[A_{i}^{\dagger} B_{i}\right]$, where we take $A_{i}^{\dagger}=\Pi_{\sigma}^{(\mu \nu)}\left(\eta_{\mu}^{i} W_{\mu}^{\dagger} \otimes \mathbb{1}_{\nu}\right)$ and $B_{i}=\left(\mathbb{1}_{\mu} \otimes \eta_{\nu}^{i *} W_{\mu}^{T}\right) \Pi_{\sigma}^{(\mu \nu)}$. Exploiting the relations (34) and (35), and using that $\operatorname{Tr}\left[\Pi_{\sigma}^{(\mu \nu)}\right]=d_{\sigma} m_{\sigma}^{(\mu \nu)}$, we obtain the bound

$$
\begin{aligned}
\langle c\rangle & \geq a_{\sigma_{0}}+\sum_{\mu, \nu, \sigma}^{\prime} a_{\sigma} m_{\sigma}^{(\mu \nu)}\left|c_{\mu} c_{\nu}\right| \\
& \equiv\langle c\rangle^{O p t} .
\end{aligned}
$$

It is straightforward to see that the choice of a covariant POVM with $\Xi=|\eta\rangle\langle\eta|$ with $|\eta\rangle$ given by (39) achieves this lower bound.

\section{F. Remarks}

Remark I Up to the constant term $a_{\sigma_{0}}$, the minimum cost (41) is simply given by the expectation value of the cost matrix (42) over the normalized vector $\mathbf{v} \equiv\left(\left|c_{\mu}\right|\right)$. Therefore the optimal input state is obtained just by finding the eigenvector corresponding to the minimum eigenvalue of the cost matrix. In other words, the optimal state for the estimation of an unknown parameter is always a superposition of maximally entangled states, with the coefficients in the superposition modulated by the particular choice of the cost function. Notice the simplification of the optimization problem provided by Theorem 2: instead of optimizing a state in the Hilbert space $\mathcal{H}=\bigoplus_{\mu \in S} \mathcal{H}_{\mu} \otimes \mathbb{C}^{m_{\mu}}$ we need only to optimize a vector in $\mathbb{R}^{|S|}$, where $|S|$ is the number of irreducible representations contained in the action of the black box.

Remark II The optimal POVM of Theorem 2 is the same optimal POVM arising from the maximum likelihood criterion 18, 19. In fact, this criterion corresponds to the particular choice of the delta cost function

$$
\begin{aligned}
c(\hat{g}, g) & =-\delta(\hat{g}, g) \\
& =-\sum_{\sigma} d_{\sigma} \chi^{\sigma}\left(\hat{g} g^{-1}\right),
\end{aligned}
$$

which is of the form (40). In other words, in the case of superpositions of maximally entangled states, the result of Theorem 2 can be viewed as the extension of the maximum likelihood approach of Ref. 18 to arbitrary cost functions.

Remark III In the optimization of covariant POVM's it is often assumed that the operator $\Xi$ corresponding to an optimal estimation can be taken with unit rank. However, for mixed states some counterexamples are known 20, 21], and for pure states there is no general proof that the POVM minimizing the average Bayes cost can be chosen with rank one. Therefore, it is important to emphasize that here the rank-one property of the optimal POVM of Theorem 2 is a result of the derivation, not an assumption.

\section{APPLICATIONS}

\section{A. Optimal transmission of reference frames}

The result of Theorem 2 can be exploited to give the definitive proof of optimality of the protocol for the absolute transmission of a Cartesian reference frame of Ref. [15], which concludes a long debate about the optimal way of communicating a reference frame [22]. Such a protocol allows two distant parties, Alice and Bob, to align their Cartesian axes in an absolute way, i.e. without the need of any kind of prior information about their relative orientations. To this purpose, Alice sends to Bob $N$ spin- $\frac{1}{2}$ particles, prepared in some fixed state. The preparation procedure of the state is related to the directions of Alice's Cartesian axes: for example Alice can align the angular momenta of some particles with her $x$-axis, some with her $y$-axis, and so on. When Bob receives the particles, since his axes are mismatched with Alice's ones, each particle appears rotated by the same unknown rotation. Then, instead of receiving the particles in the same state prepared by Alice, Bob receives them in a rotated state. Clearly, if he knows how the state should look in absence of rotations, he can try to estimate the difference, i.e. he can estimate the unknown rotation, inferring in this way the directions of Alice's axes. The precision of this scheme is defined in a Bayesian way, by taking as cost function the transmission error, i.e. the distance between the directions of Alice's axes and Bob's axes at the end of the protocol. In terms of the estimated rotation $\hat{g}$ and the true one $g$, the transmission error can be written as [15]

$$
e(\hat{g}, g)=6-2 \chi^{1}\left(\hat{g} g^{-1}\right),
$$

where $\chi^{1}(g) \equiv \operatorname{Tr}\left[U_{g}^{1}\right]$ is the character of the three dimensional irreducible representation of the rotation group. It is immediate to see that the transmission error is a cost function the form (40).

What is the best precision that can be achieved with the mentioned protocol? To answer this question we need to solve two problems: the first is to find what is the optimal state for encoding rotations, and the second is to find 
the optimal estimation strategy. It is important to stress that, since we want to achieve an absolute transmission, we are not allowed to use an external reference system, whose role would correspond to a partially shared reference frame [15]. For this reason we are allowed only to exploit the entanglement coming from the multiple equivalent representations that appear in the Clebsch-Gordan series of $U_{g}^{\otimes N}$, where $U_{g}$ is the $\mathbb{S U}(2)$ matrix that represent the rotation $g$ in the two-dimensional Hilbert space $\mathcal{H}$ of a single spin $\frac{1}{2}$ particle.

The tensor product Hilbert space $\mathcal{H}^{\otimes N}$ can be decomposed as in (4)

$$
\mathcal{H}^{\otimes N}=\bigoplus_{j=0\left(\frac{1}{2}\right)}^{\frac{N}{2}} \mathcal{H}_{j} \otimes \mathbb{C}^{m_{j}} .
$$

The irreducible representations are labeled by the quantum number $j$ of the total angular momentum, which ranges from $0\left(\frac{1}{2}\right)$ to $\frac{N}{2}$ for $N$ being even (odd), respectively. The dimension of the representation space $\mathcal{H}_{j}$ is

$$
d_{j}=2 j+1
$$

while the multiplicities are given by [15, 23]:

$$
m_{j}=\frac{2 j+1}{\frac{N}{2}+j+1}\left(\begin{array}{c}
N \\
\frac{N}{2}+j
\end{array}\right) .
$$

Since $m_{j} \geq d_{j}$ for any $j<\frac{N}{2}$, it is possible to have maximal entanglement between representation spaces and multiplicity spaces for any $j$, with the only exception of $j=\frac{N}{2}$.

However, as shown in 15, the contribution of the subspace with $j=\frac{N}{2}$ is negligible in the asymptotic limit of large $N$. Therefore we can restrict ourself to the subspace $\mathcal{H}^{\prime}=\bigoplus_{j=0\left(\frac{1}{2}\right)}^{\frac{N}{2}-1} \mathcal{H}_{j} \otimes \mathbb{C}^{m_{j}}$, and consider the state

$$
\left.|A\rangle\rangle=\bigoplus_{j=0\left(\frac{1}{2}\right)}^{\frac{N}{2}-1} \frac{c_{j}}{\sqrt{d_{j}}}\left|\mathbb{1}_{j}\right\rangle\right\rangle
$$

According to Theorem 1, and to the result of [12], this is the optimal state in the subspace $\mathcal{H}^{\prime}$ for the estimation of an unknown $\mathbb{S U}(2)$ rotation.

Now we can use Theorem 2 to state that the optimal estimation strategy is described by the covariant POVM given by $\Xi=|\eta\rangle\langle\eta|$ with

$$
\left.|\eta\rangle=\bigoplus_{j=0\left(\frac{1}{2}\right)}^{\frac{N}{2}-1} \sqrt{d_{j}}\left|\mathbb{1}_{j}\right\rangle\right\rangle
$$

The optimization of the coefficients $c_{j}$ in the state (49) has been done in [15], where the POVM (50) was assumed by exploiting for simplicity the maximum likelihood approach. In this way, the results of Theorem 11 and 2 provide the optimality proof for the protocol proposed in Ref. [15]. Therefore, we can definitely state that the asymptotic precision

$$
\langle e\rangle=\frac{8 \pi^{2}}{N^{2}}
$$

is the best that can be achieved for all input states and all POVM's, namely it is the ultimate precision limit imposed by Quantum Mechanics in the absolute alignment of two Cartesian reference frames.

\section{B. Optimal estimation of a completely unknown maximally entangled state}

Maximally entangled states are a fundamental resource for quantum teleportation [4] and for quantum cryptography 66. To achieve ideal teleportation, Alice and Bob must know with precision which maximally entangled state they are sharing, otherwise the fidelity of the state received by Bob with the original state from Alice can be lowered. Similar arguments apply to the cryptographic schemes where the correlations arising from entanglement are exploited to generate a secret key.

Now we will consider the problem of estimating in the best way a completely unknown maximally entangled state, provided that $N$ identical copies are available. This can be done as an application of Theorem 2. Let's consider a state $|\psi\rangle\rangle \in \mathcal{H} \otimes \mathcal{H}$, with $\operatorname{dim}(\mathcal{H})=d$. In terms of the notation (11), this state is maximally entangled if and only if $\psi=\frac{1}{\sqrt{d}} U$, where $U$ is some unitary operator. Using property (3), any maximally entangled state can be written as

$$
\left.\left.\left|\psi_{g}\right\rangle\right\rangle=\frac{1}{\sqrt{d}}\left(U_{g} \otimes \mathbb{1}\right)|\mathbb{1}\rangle\right\rangle,
$$

where $U_{g}$ is an element of the group $\mathbb{S U}(d)$.

If $N$ identical copies of the unknown state $\left|\psi_{g}\right\rangle$ are given, then the problem becomes to find the best estimate for parameter $g$ that labels the states of the form $\left.\left.\left|\Psi_{g}\right\rangle\right\rangle=\left|\psi_{g}\right\rangle\right\rangle^{\otimes N}$. Optimality is defined here in terms of maximization of the Uhlmann fidelity between the true state and the estimated one:

$$
f(\hat{g}, g)=\left|\left\langle\left\langle\psi_{g} \mid \psi_{\hat{g}}\right\rangle\right\rangle\right|^{2}
$$

Using the definition (52) and the property (2), we obtain

$$
f(\hat{g}, g)=\frac{1}{d^{2}}\left|\chi\left(\hat{g} g^{-1}\right)\right|^{2} .
$$

where $\chi(g)=\operatorname{Tr}\left[U_{g}\right]$. The maximization of the fidelity corresponds to the minimization of the cost function

$$
c(\hat{g}, g)=1-f(\hat{g}, g)
$$

which is of the form (40). In particular, for $d=2$, $|\chi(g)|^{2}=1+\chi^{1}(g)$, where $\chi^{1}(g)=\operatorname{Tr}\left[U_{g}^{1}\right]$ is the character 
of the irreducible representation of $\mathbb{S U}(2)$ with angular momentum $j=1$, whence we have

$$
c(\hat{g}, g)=\frac{1}{4}\left(3-\chi^{1}\left(g^{-1} \hat{g}\right)\right) .
$$

All the states of the form $\left.\left.\left|\Psi_{g}\right\rangle=\left|\psi_{g}\right\rangle\right\rangle\right\rangle^{\otimes N}$ are generated from the input state

$$
\left.|\Psi\rangle=\frac{1}{\sqrt{d^{N}}}|\mathbb{1}\rangle\right\rangle^{\otimes N}
$$

by the action of the representation

$$
\mathrm{R}(\mathbf{G})=\left\{\left(U_{g} \otimes \mathbb{1}\right)^{\otimes N} \mid U_{g} \in \mathbb{S U}(d)\right\} .
$$

Now we need to know how the input state is decomposed with respect to the invariant subspaces of this representation.

Lemma 4 Using suitable bases for the muliplicity spaces in decomposition (4), the input state (57) can be written as

$$
\left.|\Psi\rangle=\bigoplus_{\mu \in \mathrm{S}} \frac{c_{\mu}}{\sqrt{d_{\mu}}}\left|\mathbb{1}_{\mu}\right\rangle\right\rangle
$$

where the sum runs over the irreducible representations of $\mathbb{S U}(d)$ occurring in the Clebsch-Gordan series of $\mathrm{R}(\mathbf{G})$ (58), and

$$
c_{\mu}=\sqrt{\frac{d_{\mu} m_{\mu}}{d^{N}}},
$$

$d_{\mu}$ and $m_{\mu}$ being respectively the dimension and the multiplicity of the representation $\mu$ in the Clebsch-Gordan series of $\left\{U_{g}^{\otimes N}\right\}$.

Proof. See appendix.

Thank to this lemma we can exploit directly the result of Theorem 2 to calculate the average fidelity. Now we will carry on the calculation of the optimal fidelity in the simplest case $d=2$. As usual, the irreducible representations of $\mathbb{S U}(2)$ are labeled by the quantum number $j$, ranging from $0\left(\frac{1}{2}\right)$ to $\frac{N}{2}$ for $N$ being even (odd), respectively. The minimum cost can be evaluated using Theorem 2 as

$$
\langle c\rangle^{O p t}=\frac{3}{4}+\sum_{i, j=0\left(\frac{1}{2}\right)}^{\frac{N}{2}}\left|c_{i}\right| C_{i j}\left|c_{j}\right|
$$

Using Eq. (60) with the values of dimensions and multiplicities given by (47) and (48), the coefficients of the state become

$$
c_{i}=g(i) \sqrt{\frac{1}{2^{N}}\left(\begin{array}{c}
N \\
\frac{N}{2}+i
\end{array}\right)},
$$

where

$$
g(i)=\frac{2 i+1}{\sqrt{\frac{N}{2}+i+1}} .
$$

On the other hand, the matrix $C_{i j}$ is calculated according to the definition (42), namely by evaluating the multiplicity of the representation with angular momentum $k=1$ in the Clebsch-Gordan series of the tensor product $U_{g}^{i} \otimes U_{g}^{j *}$. In this way we get

$$
C_{i j}=-\frac{1}{4}\left(\delta_{i, j}+\delta_{i, j+1}+\delta_{i, j-1}\right) .
$$

Since $\sum_{i}\left|c_{i}\right|^{2}=1$, we have

$$
\langle c\rangle=\frac{1}{2}\left(1-\sum_{j=0\left(\frac{1}{2}\right)}^{\frac{N}{2}-1} c_{j} c_{j+1}\right) .
$$

To obtain the asymptotic behavior of the optimal fidelity, we can approximate the binomial distribution in (62) with a Gaussian $G_{\sigma}(x)$ with mean $\bar{x}=0$ and variance $\sigma^{2}=\frac{N}{4}$. Since the sum in (65) runs over a large interval with respect to $\sigma$, we can also approximate it with an integral over $[0,+\infty]$. All these approximations hold up to order higher than $\frac{1}{N}$. Thus the evaluation of the optimal fidelity is reduced to the evaluation of the integral

$$
I=\int_{0}^{\infty} \mathrm{d} x g(x) g(x+1) G_{\sigma}(x),
$$

whose leading order can be obtained from Taylor expansion. In this way, we derive the asymptotic cost

$$
\langle c\rangle^{O p t}=\frac{3}{4 N},
$$

corresponding to the optimal fidelity

$$
\langle f\rangle^{O p t}=1-\frac{3}{4 N} .
$$

Remarkably, the Bayes cost with uniform a priori distribution has the same asymptotic behavior of the cost of the optimal locally unbiased estimator obtained in [14, for any possible value $g$ of the true parameter. This means that in the present unbiased case the covariant measurement of Theorem 2 is optimal not only on average but also pointwise.

\section{CONCLUSIONS}

In this paper we solved the general problem of optimal estimation of group transformations in the Bayesian framework with uniform prior. For this purpose, we introduced a class of cost functions generalizing the Holevo class for phase estimation, containing the maximum likelihood strategy as a special case. For this family of cost functions, we derived the general form of the optimal input states, which involves maximal entanglement between representation and multiplicity spaces of the group action. More precisely, the form of an optimal input state is a direct sum of maximally entangled states, and for a 
given cost function one only needs to optimize the coefficients in the sum. Moreover, for any state of the optimal form all invariant cost functions lead to the same optimal $P O V M$. In this way, it is possible to derive an explicit expression for the average cost and to reduce the optimization of the state to a simple eigenvalue problem. As applications of the general result we have given the first complete derivation of the ultimate precision limit imposed by Quantum Mechanics in the absolute alignment of two Cartesian reference frames, and we have derived the optimal estimation of a completely unknown twoqubit maximally entangled state with $N$ copies of the state. In the present paper we focused attention to compact groups and finite dimensional Hilbert spaces, nevertheless an extension of our results to infinite dimensional Hilbert spaces and non-compact groups is possible, in the same way as in 19. However, since in infinite dimension the optimal states may be non-normalizable, one has to approximate them with physical states by fixing additional constraints as, typically, the energy constraint.

\section{Acknowledgments}

This work has been supported by the FET European Networks on Quantum Information and Communication Contract IST-2000-29681:ATESIT, by MIUR 2003Cofinanziamento, and by INFM PRA-2002-CLON.

\section{APPENDIX}

\section{A. Invariant cost functions}

In this section we prove the form (18) of any invariant cost function.

Proposition 1 The following integral formula holds:

$$
\int \mathrm{d} g U_{g}^{\mu} \otimes U_{g}^{\nu *}=\delta_{\mu \nu} \frac{\left.\left|\mathbb{1}_{\mu}\right\rangle\right\rangle\left\langle\left\langle\mathbb{1}_{\mu}\right|\right.}{d_{\mu}} .
$$

Proof. Using Eq. (6), we recognize in the l.h.s. the projection onto the subspace of $\mathcal{H}_{\mu} \otimes \mathcal{H}_{\nu}$ that carries the trivial representation in the Clebsch-Gordan decomposition of $U_{g}^{\mu} \otimes U_{g}^{\nu *}$. Using the orthogonality of characters, one can prove that such tensor product contains the trivial representation if and only if $\mu=\nu$. Moreover, if $\mu=\nu$, then the multiplicity of the trivial representation is one. Using the property (3), we see that the vector $\left.\left|\mathbb{1}_{\mu}\right\rangle\right\rangle$ is invariant under $U_{g}^{\mu} \otimes U_{g}^{\mu *}$. Therefore the r.h.s. is the projection onto the one-dimensional invariant subspace that carries the trivial representation, whence it coincides with the l.h.s.

Proposition 2 Any invariant function $c(\hat{g}, g)$ has the form

$$
c(\hat{g}, g)=\sum_{\mu} a_{\mu} \chi^{\mu}\left(g^{-1} \hat{g}\right)
$$

where $\chi^{\mu}(g) \equiv \operatorname{Tr}\left[U_{g}^{\mu}\right]$.

Proof. For each irreducible representation $\mu$, consider the matrix elements $u_{i j}^{\mu}(g) \equiv\left\langle\psi_{i}^{\mu}\left|U_{g}^{\mu}\right| \psi_{j}^{\mu}\right\rangle$ with respect to a fixed basis $\mathrm{B}^{\mu}=\left\{\left|\psi_{i}^{\mu}\right\rangle \mid i=1, \ldots, d_{\mu}\right\}$ for the representation space $\mathcal{H}_{\mu}$. Since the collection of all these matrix elements is an orthogonal basis for $L^{2}(\mathbf{G})$ [17, we can expand the function $c(\hat{g}, g)$ as

$$
c(\hat{g}, g)=\sum_{\mu, \nu} \sum_{i, j=1}^{d_{\mu}} \sum_{k, l=1}^{d_{\nu}} a_{i j k l}^{\mu \nu} u_{i j}^{\mu}(\hat{g}) u_{k l}^{\nu *}(g),
$$

where the complex conjugate in $u_{k l}^{\nu *}(g)$ is for later convenience. Now, the function $c$ is both left- and rightinvariant, whence it coincides with its average $\bar{c}(\hat{g}, g) \equiv$ $\int \mathrm{d} k \int \mathrm{d} h c(k \hat{g} h, k g h)$. Using Proposition 11 and Eqs. (2) and (3), we obtain

$$
\begin{aligned}
c(\hat{g}, g) & =\int \mathrm{d} k \int \mathrm{d} h c(k \hat{g} h, k g h) \\
& =\sum_{\mu, \nu} \sum_{i, j, k, l} a_{i j k l}^{\mu \nu} \delta_{\mu \nu} \times \\
& \times\left\langle\psi_{i}^{\mu}\left|\left\langle\psi_{k}^{\mu}\left|\frac{\left.\left|\mathbb{1}_{\mu}\right\rangle\right\rangle\left\langle\left\langle\mathbb{1}_{\mu}\right|\right.}{d_{\mu}}\left(U_{\hat{g}}^{\mu} \otimes U_{g}^{\mu *}\right) \frac{\left.\left|\mathbb{1}_{\mu}\right\rangle\right\rangle\left\langle\left\langle\mathbb{1}_{\mu}\right|\right.}{d_{\mu}}\right| \psi_{j}^{\mu}\right\rangle\right| \psi_{l}^{\mu}\right\rangle \\
& =\frac{1}{d_{\mu}^{2}} \sum_{\mu} \sum_{i, j, l, k} a_{i j k l}^{\mu \mu} \delta_{i k} \delta_{j l} \operatorname{Tr}\left[U_{\hat{g} g^{-1}}^{\mu}\right] \\
& =\sum_{\mu} a_{\mu} \chi^{\mu}\left(g^{-1} \hat{g}\right),
\end{aligned}
$$

where $a_{\mu} \equiv \frac{1}{d_{\mu}^{2}} \sum_{i, j} a_{i j i j}^{\mu \mu}$.

\section{B. Decomposition of a product of maximally entangled states}

Here we give the proof for Lemma 4

Proof. Consider the representation $\mathrm{R}(\mathbf{G})=\left\{\left(U_{g} \otimes\right.\right.$ $\left.\mathbb{1})^{\otimes N} \mid U_{g} \in \mathbb{S U}(d)\right\}$. It is convenient to order the $2 N$ Hilbert spaces in the tensor product $\mathcal{H}^{\otimes 2 N}$ in such a way that the unitary operators act on the first $N$ spaces, while the identity operators acts on the second $N$ spaces. With this ordering, by defining $\mathcal{H}_{A}\left(\mathcal{H}_{B}\right)$ the tensor product of the first (second) $N$ spaces, we have $\mathrm{R}(\mathbf{G})=\mathrm{R}_{A}(\mathbf{G}) \otimes \mathbb{1}_{B}$, where $\mathbf{R}_{A}(\mathbf{G}) \equiv\left\{U_{g}^{\otimes N} \mid U_{g} \in \mathbb{S U}(d)\right\}$ is the $N$-fold tensor representation of $\mathbb{S U}(d)$.

Let's decompose now the Hilbert space $\mathcal{H}_{A}$ with respect to the action of the representation $\mathrm{R}_{A}(\mathbf{G})$ :

$$
\mathcal{H}_{A}=\bigoplus_{\mu} \mathcal{H}_{\mu} \otimes \mathbb{C}^{m_{\mu}}
$$

The tensor product $\mathcal{H}_{A} \otimes \mathcal{H}_{B}$ can be decomposed with respect to $\mathrm{R}(\mathbf{G})=\mathrm{R}_{A}(\mathbf{G}) \otimes \mathbb{1}_{B}$ as

$$
\mathcal{H}_{A} \otimes \mathcal{H}_{B}=\bigoplus_{\mu} \mathcal{H}_{\mu} \otimes \mathbb{C}^{M_{\mu}}
$$


where the multiplicity has been increased to $M_{\mu}=$ $m_{\mu} \times d^{N}$, since $\mathcal{H}_{B}$ has been absorbed in the multiplicity spaces.

With respect to the factorization $\mathcal{H}^{\otimes 2 N}=\mathcal{H}_{A} \otimes \mathcal{H}_{B}$, the input state $|\Psi\rangle=|\mathbb{1}\rangle\rangle^{\otimes N}$ can be written as

$$
\left.|\Psi\rangle=\frac{1}{\sqrt{d^{N}}}\left|\mathbb{1}^{\otimes N}\right\rangle\right\rangle,
$$

where $\mathbb{1}^{\otimes N}$ is the identity in $\mathcal{H}_{A} \equiv \mathcal{H}^{\otimes N} \equiv \mathcal{H}_{B}$. Here we are using notation (11), with respect to the product basis $\mathrm{B}^{\otimes N}$ for $\mathcal{H}_{A}$ and $\mathcal{H}_{B}$, B being a fixed basis for $\mathcal{H}$. Now we want to change the basis in $\mathcal{H}_{A}$, by switching from $\mathrm{B}^{\otimes N}$ to $\mathrm{B}^{\prime} \equiv \bigcup_{\mu} \mathrm{B}_{R}^{\mu} \otimes \mathrm{B}_{M}^{\mu}$, where $\mathrm{B}_{R}^{\mu} \equiv\left\{\left|\psi_{n}^{\mu}\right\rangle \mid n=\right.$ $\left.1, \ldots, d_{\mu}\right\}\left(\mathrm{B}_{M}^{\mu} \equiv\left\{\left|\phi_{n}^{\mu}\right\rangle \mid n=1, \ldots, m_{\mu}\right\}\right)$ is a basis for the representation (multiplicity) space in Eq. (72). One has

$$
\begin{aligned}
|\Psi\rangle & =\frac{1}{\sqrt{d^{N}}} \bigoplus_{\mu} \sum_{m=1}^{d_{\mu}} \sum_{n=1}^{m_{\mu}}\left|\psi_{m}^{\mu}\right\rangle_{A}\left|\phi_{n}^{\mu}\right\rangle_{A}\left|\psi_{m}^{\mu *}\right\rangle_{B}\left|\phi_{n}^{\mu *}\right\rangle_{B} \\
& =\frac{1}{\sqrt{d^{N}}} \bigoplus_{\mu} \sum_{m=1}^{d_{\mu}} \sqrt{m_{\mu}} \underline{\left|\psi_{m}^{\mu}\right\rangle\left|\tau_{m}^{\mu}\right\rangle,}
\end{aligned}
$$

[1] I. L. Chuang and M. A. Nielsen, Quantum Information and Quantum Computation, Cambridge University Press (Cambridge, UK, 2000)

[2] P. W. Shor, p. 124 in Proceedings pf the 35th Annual Symposium of the Foundations of Computer Science, ed. S. Goldwasser (IEEE Computer Society Press, Los Alamitos, CA, 1994).

[3] L. K. Grover, p. 212 in Proceedings, 28th Annual ACM Symposium on the Theory of Computing (STOC), (May 1996), preprint quant-ph/9605043; Phys. Rev. Lett. 79, 325 (1997).

[4] C. H. Bennett, G. Brassard, C. Crepeau, R. Jozsa, A. Peres, and W. K. Wootters, Phys. Rev. Lett. 70, 1895 (1993).

[5] C. H. Bennett and S. J. Wiesner, Phys. Rev. Lett. 69, 2881 (1992).

[6] A. K. Ekert, Phys. Rev. Lett. 67, 661 (1991); Nature 358, 14 (1992).

[7] G. M. D'Ariano, P. Lo Presti, and M. G. A. Paris, Phys. Rev. Lett. 87, 270404 (2001).

[8] V. Giovannetti, S. Lloyd, and L. Maccone, Science 306, 1330 (2004).

[9] C. W. Helstrom, Quantum detection and estimation theory (Academic Press, New York, 1976).

[10] A. S. Holevo, Probabilistic and Statistical Aspects of Quantum Theory (North Holland, Amsterdam, 1982).

[11] A. Acín, Phys. Rev. Lett. 87, 177901 (2001).

[12] A. Acín, E. Jane, and G. Vidal, Phys. Rev. A 64, where we defined the normalized vector

$$
\left|\tau_{m}^{\mu}\right\rangle \equiv \frac{1}{\sqrt{m_{\mu}}} \sum_{n=1}^{m_{\mu}}\left|\phi_{n}^{\mu}\right\rangle\left|\psi_{n}^{\mu *}\right\rangle\left|\phi_{n}^{\mu *}\right\rangle
$$

Therefore, exploiting notation (11) with respect to the bases $\left\{\left|\psi_{m}^{\mu}\right\rangle\right\}$ and $\left\{\left|\tau_{m}^{\mu}\right\rangle\right\}$, we can write

$$
\left.|\Psi\rangle=\bigoplus_{\mu} \sqrt{\frac{m_{\mu}}{d^{N}}}\left|\mathbb{1}_{\mu}\right\rangle\right\rangle
$$

050302(R) (2001).

[13] A. Fujiwara, Phys. Rev. A 65, 012316 (2001).

[14] M. A. Ballester, Phys. Rev. A 69, 022303 (2004).

[15] G. Chiribella, G. M. D'Ariano, P. Perinotti, and M. F. Sacchi, Phys. Rev. Lett. 93, 180503 (2004).

[16] G. M. D'Ariano, P. Lo Presti, and M. F. Sacchi, Phys. Lett. A 272, 32 (2000).

[17] See, for example, Chapter 7 of A. O. Barut and R. Raczka, Theory of Group Representations and Applications (World Scientific, 1986).

[18] G. Chiribella, G. M. D'Ariano, P. Perinotti, and M. F. Sacchi, Phys. Rev. A 70, 062105 (2004).

[19] G. Chiribella, G. M. D'Ariano, P. Perinotti, and M. Sacchi, Int. J. Quant. Inf. (in press).

[20] G. Chiribella and G. M. D'Ariano, J. Math. Phys. 45, 4435 (2004).

[21] G. M. D'Ariano, C. Macchiavello, and P. Perinotti, quant-ph/0411133.

[22] A. Peres and P. F. Scudo, Phys. Rev. Lett. 87, 167901 (2001); E. Bagan, M. Baig, and R. Muñoz-Tapia, Phys. Rev. Lett. 87, 257903 (2001); A. Peres and P. F. Scudo, J. Mod. Opt. 49, 1235 (2002); E. Bagan, M. Baig, and R. Muñoz-Tapia, Phys. Rev. A 70, 030301 (2004); E. Bagan, M. Baig, and R. Muñoz-Tapia, Phys. Rev. A 70, 030301(R) (2004); M. Hayashi, quant-ph/0407053.

[23] J. I. Cirac, A. K. Ekert, and C. Macchiavello, Phys. Rev. Lett. 82, 4344 (1999). 